\title{
Mechanics Performance Analysis of New Quasi-Honeycomb Sandwich Structure's Core
}

\author{
Huanghai Xie \\ College of Mechanical and Power Engineering \\ China Three Gorges University \\ Yichang 443002, China \\ huanghxie@163.com \\ Yixian Du \\ College of Mechanical and Power Engineering \\ China Three Gorges University \\ Yichang 443002, China \\ duyixian@aliyun.com \\ Xiang Li \\ College of Mechanical and Power Engineering \\ China Three Gorges University \\ Yichang 443002, China \\ lixiangcfy@163.com
}

\author{
Guan Tong \\ College of Mechanical and Power Engineering \\ China Three Gorges University \\ Yichang 443002, China \\ icecooker@suhu.com \\ Jinxue Wang \\ China Three Gorges University \\ Yichang 443002, China \\ sdwjx2009@163.com \\ Shuangqiao Yan \\ College of Mechanical and Power Engineering \\ China Three Gorges University \\ Yichang 443002, China \\ yan_s_q@163.com
}

\begin{abstract}
At present the study of mechanical properties of new quasi-honeycomb sandwich structure's core research, ignored the thickness of quasi-honeycomb cell wall,that is, when establish finite element model of the structure, pick up the same thickness of quasi-honeycomb cell wall of modeling; While in practice, the hexagonal hypotenuse of the quasi-honeycomb unit cell should be double. In response to this problem, in consideration of the unit cell thickness conditions, re-derive the effective elastic constants of new quasi-honeycomb sandwich structure's core and compared with those of hexagonal honeycomb sandwich structure, and the results show that both equivalent elastic moduli are approximately equal, but the quasi-honeycomb sandwich structure is endowed with higher equivalent shear modulus and lower equivalent body density, thus the total structure mass can be effectively reduced.
\end{abstract}

Keywords-Sandwich structure; Quasi-honeycomb; equivalent elastic constants; numerical simulation.

\section{INTRODUCTION}

With the rapid development of today's industrial equipment technology (such as high-grade numerical control machine) and transportation (e.g., aerospace aircraft, high-speed locomotives, ships), the design and preparation for high performance engineering materials has been put forward higher requirements, such as: light weight, high strength, high toughness, more functional applications, etc.[1-4] In addition, as it depends on the development of new typed high-precision manufacturing technology, the manufacturing cost is fairly high. In this case, to improve the design or innovate the configuration of the present matured honeycomb sandwich structure's core is of significant innovation and engineering application value. Therefore, Dr. Li Xiang, from the perspective of bionics and innovation of configuration, raises the concept of new quasi-honeycomb sandwich structure[5], proposed that by optimizing the arrangement of hexagon and trapezium unit cell, to construct a brand new new quasi-honeycomb sandwich structure[6-10].

\section{MECHANICAL EQUIVALENT MODEL OF QUASI-HONEYCOMB SANDWICH STRUCTURES}

According to the Gibson's unit cell theory,selecting the most basic hexagon and trapezium composition unit of honeycomb sandwich as the cell unit. The structure and geometry relations of the cell unit (later referred to as unit cell) are shown in figure 1. 


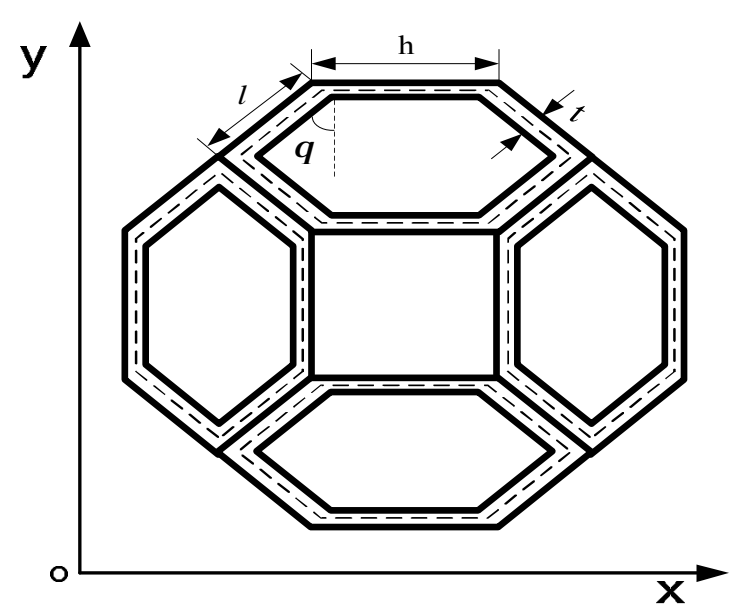

Fig.1. Structure and physical dimension of quasi-honeycomb sandwich unit cell

To analyze its equivalent mechanical performance, the simplified model of cell unit is built[5], as is shown in figure 1 , under the premise of considering the flexibility of the cell wall. The thickness of $l$ equals $2 t$.Using force balance principle, the material mechanics beam bending theory and knowledge of the conservation of mass, the equivalent mechanical parameters of the honeycomb sandwich layer are presented, as is shown in figure 2 .

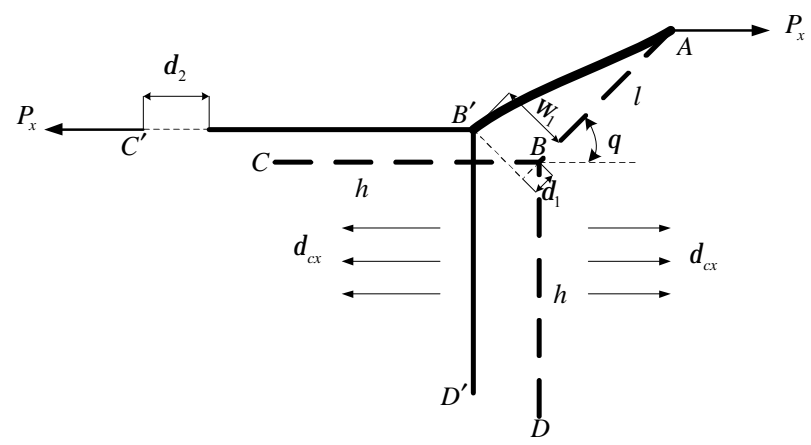

(a)X-direction tensile

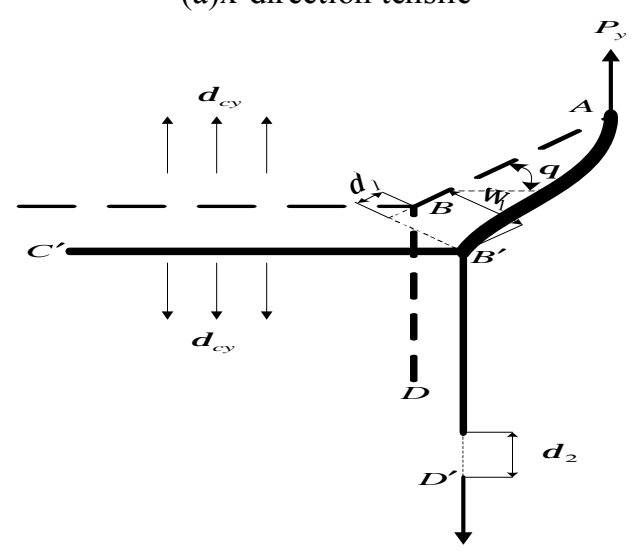

(b)y-direction tensile

Fig.2 Force diagram of part cell of quasi-honeycomb core

\section{SANDWICH EQUIVALENT ELASTIC CONSTANTS IN X DIRECTION DERIVATION}

As shown in Figure 1(a), according to the force equilibrium conditions:

$$
\begin{aligned}
& M=\frac{1}{2} P_{x} l \sin \theta \\
& P_{x}=\sigma_{c x} A_{x}=\sigma_{c x}(h+l \sin \theta) b
\end{aligned}
$$

$M$ —node bending moment of quasi-honeycomb core, $\mathrm{N} \cdot \mathrm{m}$;

$P_{x}$-external force of quasi-honeycomb core, $\mathrm{N}$;

$A_{x}$ - sectional area of quasi-honeycomb core in $\mathrm{x}$ direction derivation, $\mathrm{m}^{2}$;

$b$-height of quasi-honeycomb core, $\mathrm{m}$. $\mathrm{AB}:$

according to beam flexure theory, the flexibility of

$$
w_{1}=\frac{P_{x} l^{3} \sin \theta}{12 E_{s} I}
$$

let $\quad I=\frac{1}{12} b(2 t)^{3} \quad$ (inertia moment) into equation(3):

$$
w_{1}=\frac{P_{x} l^{3} \sin \theta}{8 E_{s} b t^{3}}
$$

according to Hooke's Law,extensional capacity of panel AB is:

$$
\begin{aligned}
& \delta_{1}=\varepsilon_{A B}^{x} l=\frac{\sigma_{A B}^{x}}{E_{s}} l=\frac{P_{x} \cos \theta}{2 b t} \frac{l}{E_{s}} \\
& \varepsilon_{A B}^{x}=\frac{\sigma_{A B}^{x}}{E_{s}} \text { is the line strain of panel } \mathrm{AB},
\end{aligned}
$$$$
\sigma_{A B}^{x}=\frac{P_{x} \cos \theta}{2 b t} \text { is the positive stress. }
$$

Simultaneously, extensional capacity of panel BC is:

$$
\delta_{2}=\varepsilon_{B C}^{x} h=\frac{\sigma_{B C}^{x}}{E_{s}} l=\frac{P_{x}}{b t} \frac{h}{E_{s}}
$$

According to Hooke's Law,equivalent strain $\varepsilon_{c x}$ in $\mathrm{x}$ direction derivation:

$$
\varepsilon_{c x}=\frac{\Delta l_{x}}{l_{x}}=\frac{w_{1} \sin \theta+\delta_{1} \cos \theta+\delta_{2}}{l \cos \theta+h}
$$

Let equation (4),(5) into equation (7):

$$
\varepsilon_{c x}=\frac{P_{x}\left(l^{3} \sin ^{2} \theta+4 t^{2} l \cos ^{2} \theta+8 t^{2} h\right)}{8 E_{s} b t^{3}(l \cos \theta+h)}
$$

Simultaneously, equivalent $\operatorname{strain} \varepsilon_{c y}$ in $\mathrm{y}$ direction derivation:

$\varepsilon_{c y}=\frac{\Delta l_{y}}{l_{y}}=\frac{w_{1} \cos \theta-\delta_{1} \sin \theta}{h+l \sin \theta}=\frac{P_{x} \sin \theta \cos \theta l\left(l^{2}-4 t^{2}\right)}{8 E_{s} b(h+l \sin \theta) t^{3}}$

(9) 
According to the definition of Poisson's ratios, equivalent Poisson's ratios of quasi-honeycomb core in $\mathrm{x}$ direction derivation $\mathrm{V}_{c x}$ :

$$
v_{c x}=\left|\frac{\varepsilon_{c y}}{\varepsilon_{c x}}\right|=\frac{\varepsilon_{c}}{\varepsilon_{c x}}=\frac{\cos \theta+\beta}{\sin \theta+\beta} \times \frac{\sin \theta \cos \theta\left(1-4^{2} / l^{2}\right)}{\sin ^{2} \theta+\cos ^{2} \theta \times 4 t^{2} / l^{2}+\beta \times 8 t^{2} / l^{2}}
$$

where $\beta=h / l$

According to the definition of elastic modulus, equivalent elastic modulus of $\mathrm{x}$ direction of hexagonal cell of honeycomb sandwich core $E_{c x}$ :

$$
E_{c x}=\frac{\sigma_{c x}}{\varepsilon_{c x}}=E_{s} \frac{8^{3}}{l^{3}} \frac{\cos \theta+\beta}{\sin \theta+\beta} \times \frac{1}{\sin ^{2} \theta+\cos ^{2} \theta \times 4 t^{2} / l^{2}+\beta \times \phi^{2} / l^{2}}
$$

Simultaneously, equivalent Poisson's ratios of quasi-honeycomb core in y direction derivation $\mathrm{v}_{c y}$ :

$$
v_{c}=\frac{\varepsilon_{\alpha}}{\varepsilon_{c}} \mid=\frac{\varepsilon_{\alpha}}{\varepsilon_{c}}=\frac{\sin \theta+\beta}{\cos \theta+\beta} \times \frac{\sin \theta \cos \theta\left(1-\boldsymbol{t}^{2} / l^{2}\right)}{\left(\cos ^{2} \theta+\sin ^{2} \theta \times \boldsymbol{t}^{2} / l^{2}+\beta \times 8^{2} / l^{2}\right)}
$$

$$
E_{c y}=\frac{\sigma_{c y}}{\varepsilon_{c y}}=8 E_{s} \frac{t^{3}}{l^{3}} \frac{\sin \theta+\beta}{\cos \theta+\beta} \times \frac{1}{\cos ^{2} \theta+\sin ^{2} \theta \times 4 t^{2} / l^{2}+\beta \times 8 t^{2} / l^{2}}
$$

equivalent elastic modulus of y direction of hexagonal cell of honeycomb sandwich core $E_{c y}$ :

where $\beta=h / l, t$-thickness of the core, $h$-width of hexagonal cell of honeycomb sandwich core, $l$-the short edge length of hexagonal cell of honeycomb sandwich core, $\theta$-angle between the hexagonal short side and the horizontal side, $E_{s}$-elastic (Young's) modulus of core's material, $E_{c x}$-equivalent elastic modulus of $\mathrm{X}$ direction of hexagonal cell of honeycomb sandwich core, $E_{c y}$-equivalent elastic modulus of $\mathrm{Y}$ direction of hexagonal cell of honeycomb sandwich core, $G_{c x y}$ - equivalent shear modulus of core on the XY plane, $v_{c x}, \quad v_{c y}$ - equivalent Poisson's ratios of core.

The sandwich panel's sandwich is aluminum honeycomb, made of 2024 aluminum alloy material. Its yield strength is $758 \mathrm{MPa}$, density is $\rho_{s}=2780 \mathrm{~kg} / \mathrm{m}^{3}$, other data is shown in table 1.

Table 1 : Size parameters of quasi-honeycomb sandwich panel for the structure

\begin{tabular}{|c|c|c|c|}
\hline \multirow{2}{*}{$\begin{array}{c}\text { Cell size of } \\
\text { core }(\mathrm{mm})\end{array}$} & $t$ & $l$ & $h$ \\
\cline { 2 - 4 } & 0.05 & 5 & 10 \\
\hline $\begin{array}{c}\text { Sandwich } \\
\text { material(aluminum) }\end{array}$ & $E_{s}(\mathrm{GPa})$ & $\mathrm{v}_{s}$ & $\rho_{s}$ \\
\cline { 2 - 4 } & 70 & 0.26 & $2780 \mathrm{~kg} / \mathrm{m}^{3}$ \\
\hline
\end{tabular}

According to the formula (14) we can obtain the equivalent elastic constants of the aluminum quasi-honeycomb,and compare with the honeycomb, shown in table 2

\begin{tabular}{|l|c|c|c|c|c|}
\hline & $E_{c x}(\mathrm{MPa})$ & $\begin{array}{c}E_{c y} \\
(\mathrm{MPa})\end{array}$ & $\mathrm{v}_{c x}$ & $\mathrm{v}_{c y}$ & $\begin{array}{c}G_{c x y}(\mathrm{M} \\
\mathrm{Pa})\end{array}$ \\
\hline New & 1.159 & 1.159 & 0.996 & 0.996 & 0.0134 \\
\hline Old & 0.162 & 0.162 & 0.999 & 0.999 & 0.0404 \\
\hline
\end{tabular}

Table 2: Comparison between honeycomb and quasi-honeycomb

\section{CONCLUSIONS}

The study presented in this paper is aimed at the equivalent mechanical properties of quasi-honeycomb core. Using sandwich plate theory and the Gibson formulas, in consideration of the unit cell thickness conditions, re-derive the effective elastic constants of new quasi-honeycomb sandwich structure's core,compared with those of hexagonal honeycomb sandwich structure. The results show that the mechanical properties of the sandwich structure of the new quasi-honeycomb superior mechanical properties of conventional honeycomb sandwich structure.

\section{ACKNOWLEDGEMENTS}

This research was partially funded by National Natural Science Foundation of China:"Study of topology optimization method for compliant mechanism using meshless method considering fluid-structural interaction"(51105229);

"Innovating configuration and integrated design method of structure-material properties for ultralight and porous class honeycomb sandwich structure material"(51305232),

Hubei Provincial Natural Science Foundation (2013C FB222),2014 Annual Graduate Research Innovation Fund of China Three Gorges University.

\section{REFERENCES}

[1]. Xiang Li, Gangyan Li, Chun H. Wang and Min You. Minimum-Weight Sandwich Structure Optimum Design Subjected to Torsional Loading [J]. Applied Composite Materials, 19(2012) 117-126.

[2]. Xiang Li, Gangyan Li, Chun H. Wang and Min You. Optimum design of composite sandwich structures subjected to combined torsion and bending loads $[\mathrm{J}]$. International of Applied Composite Materials, 19(2012) 315-331.

[3]. Xiang Li, Gangyan Li, Chun H. Wang and Min You. 
Minimum-Weight Sandwich Structure Optimum Design Subjected to Torsional Loading[J]. Applied Composite Materials, 2012, 19:117-126.(DOI 10.1007/s10443-010-9185-4)

[4]. LI Xiang, YU Licheng, Zhou youhui, Li Yang, Zhang Xun. Numerical simulation of new quasi-honeycomb sandwich structure's core. Advanced Materials Research Vols. 834-836 (2014) pp 1601-1606

[5] Gibson, L. J. and Ashby, M. F. Cellular solids: structure and properties[M]. Cambridge [u.a.]: Cambridge Univ. Press, 1999.

[6]. Xiang Li,Li Gangyan, You Min. Research on Simulation of Car Information Centralized Control System [J]. Journal of China Three Gorges Univ.(Natural Sciences),2008,30(6).

[7]. Xiang Li, Gangyan Li and Chun H. Wang. Optimisation of Composite Sandwich Structures Subjected to Combined Torsion and Bending Stiffness Requirements[J]. Applied Composite Materials, In Press, Published online: 19 July 2011, p. 1-16. (DOI 10.1007/s10443-011-9221-z).

[8]. Li Xiang, You Min. Mechanics performance analysis and numerical simulation of sandwich structure's core for satellite [J]. Journal of China Three Gorges Univ.(Natural Sciences),2012,34(4).

[9]. Li Xiang. Sandwich structure Lightweight Design Subjected to Multiple Loading constraints and Its Application[D]. Wuhan University of Technology, 2011.

[10]. WEI Jing, LI Zhen, SUN Wei, et al. Shape and topology optimization for gearbos of high speed train based on SIMP model and strain energy theory[J]. Journal of Mechanical Strength, 2011, 33(4): 558-564 